\title{
VARIABILIDADE TEMPORAL E ESPACIAL DA PRECIPITAÇÃO PLUVIOMÉTRICA EM PERNAMBUCO ATRAVÉS DE ÍNDICES DE EXTREMOS CLIMÁTICOS
}

\author{
RANYÉRE SILVA NÓBREGA ${ }^{1}$, RAFHAEL FHELIPE DE LIMA FARIAS ${ }^{1}$, CARLOS ANTONIO COSTA \\ DOS SANTOS ${ }^{2}$
}

\author{
${ }^{1}$ Universidade Federal de Pernambuco, Departamento de Geografia, Recife, PE, Brasil \\ ${ }^{2}$ Universidade Federal de Campina Grande, Unidade Acadêmica de Ciências Atmosféricas, \\ Campina Grande, PB, Brasil
}

ranyere.nobrega@yahoo.com.br, rafhaelfarias@hotmail.com, carlostorm@gmail.com

Recebido Abril de 2013 - Aceito Setembro de 2014

\begin{abstract}
RESUMO
O presente artigo tem como objetivo analisar a tendência espacial e temporal da precipitação pluviométrica do Estado de Pernambuco, tendo como base índices de extremos climáticos e a técnica dos quantis. Os dados de precipitação pluviométrica diária foram oriundos do Climate Prediction Center (CPC) relativos ao período de 1978 a 2010. Para analisar a tendência da precipitação foram escolhidos os índices climáticos sugeridos pelo Expert Team on Climate Change Detection Monitoring and Indices (ETCCDMI). Através do método de krigagem foi possível analisar a variabilidade espacial dos índices analisados. Os resultados demonstram predominância de tendência de aumento nos padrões pluviométricos em grande parte do Estado, com exceção do setor central da Região Metropolitana leste e parte central da Zona da Mata. Os resultados dos índices conjuntamente indicam que as chuvas estão cada vez mais concentradas em poucos dias ao longo do ano nos sertões e agreste de Pernambuco. Também sobre o sertão, foram encontrados os maiores números de episódios extremamente secos, assim como, a maior quantidade de ocorrência de eventos extremamente chuvosos, concentrados nos meses dezembro, janeiro e fevereiro, período chuvoso do Sertão de Pernambuco.
\end{abstract}

Palavras-chave: índices climáticos; quantis; seca; eventos extremos.

\begin{abstract}
TEMPORAL AND SPATIAL VARIABILITY OF RAINFALL IN PERNAMBUCO BY EXTREME CLIMATIC INDICES

This article aims to analyze the spatial and temporal rainfall trends in Pernambuco State based on extreme climatic indices and quantile technique. The daily rainfall data are from the Climate Prediction Center (CPC) for the period 1978 to 2010. To analyze the trend in precipitation climatic indices, suggested by the Expert Team on Climate Change Detection Monitoring and Indices (ETCCDMI), were chosen. Using the kriging method it was possible to analyze the spatial variability of the indexes. The results show a predominant tendency of increasing rainfall patterns in much of the State, except for the middle region of the eastern metropolitan region and the middle region of the Zona da Mata. The overall indice results indicate that rain is becoming more concentrated in a few days throughout the year in the Sertão and Agreste regions in Pernambuco. In the Sertão of Pernambuco, the largest number of extremely dry episodes have occurred and also the occurrence of greater number of extremely rainy events, concentrated on December, January and February months, the rainy season in the Sertão region of Pernambuco.
\end{abstract}

Keywords: climatic index, quantiles, drought, extreme events. 


\section{INTRODUÇÃO}

Diversos estudos vêm diagnosticando progressivo aumento na concentração dos GEE (Gases de Efeito Estufa) na atmosfera, e há indícios que este aumento está relacionado principalmente pelas atividades antrópicas (Costa e Foley, 2000; Nijssen et al. 2001), de maneira que este aumento irá refletir diretamente no balanço de radiação de ondas longas e curtas, afetando o clima do planeta. Segundo Marengo (2006) o impacto de mudanças climáticas sobre os recursos hídricos no Brasil deverá ser mais dramático, porém no Nordeste, onde há escassez de água, já é um problema. Atualmente, a disponibilidade hídrica per capita na região é insuficiente nos Estados do Rio Grande do Norte, Paraíba, Pernambuco, Alagoas e Sergipe, sem contar as variações regionais, que tornam a situação ainda mais insustentável para os oito milhões de habitantes do semiárido.

Procurando identificar mudanças climáticas regionais, Hastenrath e Greischar (1993) examinaram séries históricas de precipitação em uma rede de estações bem distribuídas espacialmente sobre o Nordeste do Brasil (NEB). Os autores não encontraram tendências significativas para condições mais úmidas ou secas na região. No entanto, Haylock et al. (2006) recentemente analisaram a precipitação sobre a América do Sul e observaram uma tendência de aumento do total anual de chuva sobre o NEB. O estudo realizado por Santos e Brito (2007), utilizando a metodologia adotada por Haylock et al. (2006), também mostra tendência de aumento da precipitação total anual na região Nordeste, especificamente para os Estados do Rio Grande do Norte e Paraíba.

Por décadas, muitos dos estudos de variabilidade e mudanças climáticas da precipitação estiveram focados em mudanças nos valores médios. Entretanto, há uma necessidade de se verificar mudanças não só em totais pluviométricos, mas também no comportamento médio deste elemento climático, como por exemplo, ocorrência de eventos extremos de seca e/ou de chuva. Em geral, estudos de mudanças climáticas globais com base em dados meteorológicos são realizados por meio de análises de séries históricas de elementos climáticos. Baseando-se em tendências destas variáveis para um determinado local é referido se ocorreram mudanças no clima. Entretanto, para melhor definir esta questão, a Organização Mundial de Meteorologia criou um grupo de trabalho que elaborou índices de detecção de mudanças climáticas. Ao todo foram definidos 27 (vinte e sete) índices, sendo que alguns destes são aplicados para latitudes médias, outros para os trópicos e parte deles são válidos para qualquer local. Destes índices 11 são para precipitação e 16 da temperatura do ar.

Eventos extremos de precipitação, como secas severas ou grandes enchentes, alteram consideravelmente as características habituais de uma dada região, podendo causar grandes transtornos para população, seja nos grandes centros urbanos, com inundações, como também estiagens prolongadas no sertão. A partir do exposto acima, o principal objetivo deste trabalho é evidenciar possíveis mudanças temporais e espaciais no comportamento da precipitação nas últimas décadas em Pernambuco através das análises de índices de extremos climáticos dependentes de dados de precipitação diária.

\section{MATERIAL E MÉTODOS}

\section{1 Área de estudo}

Os dados de precipitação utilizados foram obtidos no Climate Prediction Center (CPC Disponível em http://cccma. seos.uvic.ca/ETCCDMI/software.shtml) da National Oceanic and Atmospheric Administrantion (NOAA). São dados diários referentes ao período de 1979 a 2010, totalizando 32 anos e consistem de informações de estações meteorológicas e plataforma de coleta de dados (PCD) interpolados para uma grade de $1^{\circ}$ grau de latitude por $1^{\circ}$ de longitude, utilizando o esquema modificado de Cressman (Glahn et al., 1985; Charba et al., 1992). Alguns autores vêm utilizando estes dados ao longo da América do Sul para análise espaço-temporal de modo mais prático e com menos falhas do que os obtidos em estações meteorológicas (Silva et al, 2007; Nóbrega et al, 2010). A principal vantagem em utilizá-los é que já estão livres de falhas, o que é muito comum quando se utiliza dados diários, tornando-os uma opção evidentemente interessante para estudos climatológicos.

Foram extraídos 8 pontos de grade dos dados de precipitação (Figura 1). O ponto 1 está localizado na área metropolitana de Recife, os pontos 2 (a e b) são localizados na mesorregião do agreste pernambucano, os pontos 3 ( $a, b$ e c) são localizados na mesorregião do sertão pernambucano e os pontos 4 (a e b) são localizados na mesorregião do sertão do São Francisco pernambucano.

\section{2 Índices climáticos}

Os índices de extremos climáticos utilizados na presente pesquisa foram sugeridos pela Equipe de Especialista em Detecção, Monitoramento e Índices de Mudanças Climáticas (Expert Team on Climate Change Detection, Monitoring and Índices - ETCCDMI). Estes índices são utilizados para estudos de extremos climáticos, baseados em dados diários de temperatura e precipitação. A equipe do ETCCDMI sugere 27 índices climáticos, sendo 16 referentes a precipitação e 11 referentes a precipitação. Neste trabalho foram utilizados apenas 8 índices de precipitação (Tabela 1) que melhor representam as características climáticas da região estudada. 


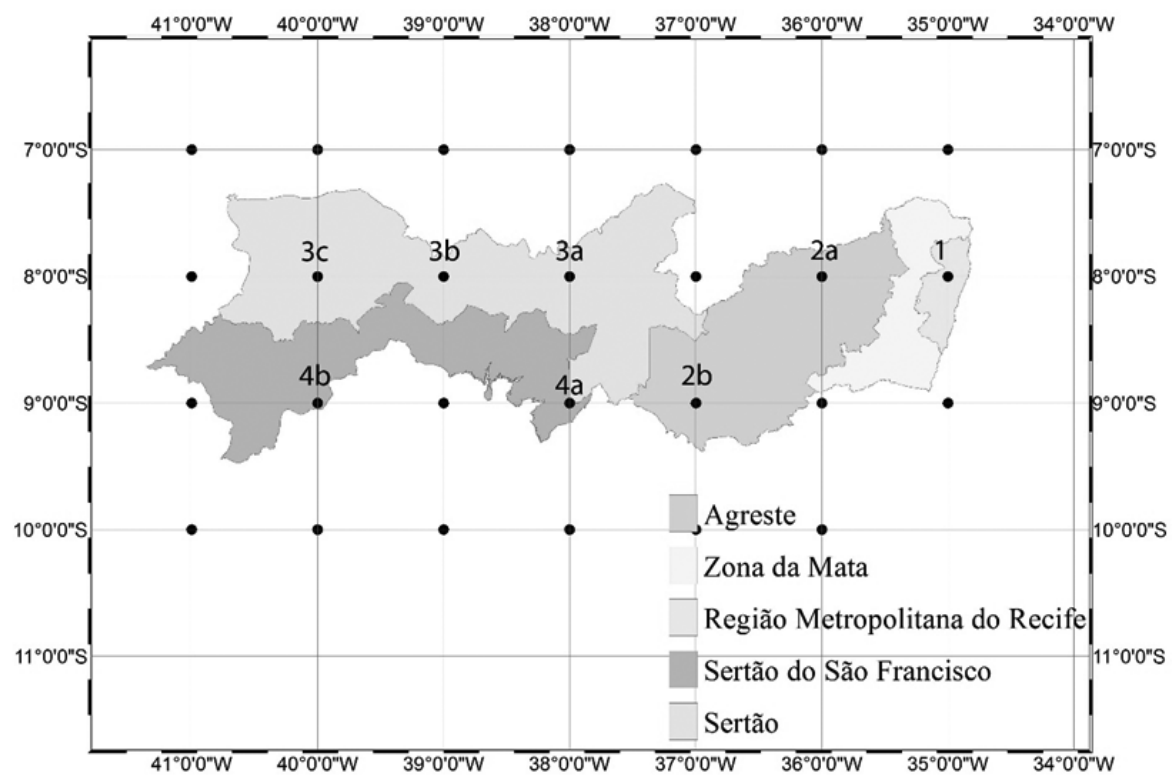

Figura 1 - Distribuição em pontos de grade e localização dos pontos analisados.

Tabela 1 - Índices de extremos climáticos baseados em dados de precipitação utilizados neste estudo.

\begin{tabular}{|l|l|c|}
\hline ÍNDICES & DEFINIÇÃO & UNIDADES \\
\hline Rx1dia & Quantidade máxima de precipitação em um dia & $\mathrm{mm} /$ dia \\
\hline Rx5dias & $\begin{array}{l}\text { Quantidade máxima de precipitação em cinco dias } \\
\text { consecutivos }\end{array}$ & $\mathrm{mm} / \mathrm{dia}$ \\
\hline R50mm & $\begin{array}{l}\text { Número de dias, em um ano, com precipitação superior à 50 } \\
\text { mm }\end{array}$ & dias/ano \\
\hline R95p & Dias muito chuvosos anuais & dias/ano \\
\hline R99p & Dias extremamente chuvosos anuais & dias/ano \\
\hline DCC & Dias Consecutivos Chuvosos anuais & dias/ano \\
\hline DCS & Dias Consecutivos Secos anuais & $\mathrm{dias} / \mathrm{ano}$ \\
\hline PRCPTOT & Precipitação Total Anual & \\
\hline
\end{tabular}

Para o cálculo dos índices optou-se por utilizar o pacote computacional RClimdex (Zhang e Yang, 2004). Os resultados gerados na saída do RClimDex fornecem análises estatísticas com tendência linear calculada pelo método de mínimos quadrados, nível de significância estatística da tendência (valor $\mathrm{p}$ ) obtido através do método de Fisher, onde estes foram definidos como alta $(p \leq 0,05)$ e boa $(p \leq 0,10)$ significância estatísticas, coeficiente de determinação $\left(\mathrm{r}^{2}\right)$ e erro padrão de estimativa, assim como os gráficos das séries anuais.

Para a análise geoespacial foi utilizado o método da krigagem, que se baseia em funções matemáticas para acrescentar pesos maiores nas posições mais próximas aos pontos amostrais e pesos menores nas posições mais distantes, e criar assim os pontos interpolados com base nessas combinações lineares. Para que o método produzisse a melhor estimativa linear, não viciada dos dados de um atributo em um local não amostrado, foi utilizado semivariograma, com efeito pepita $=$ 5,97 , alcance $=108,8$ e soleira $=10,4$ (mais detalhes sobre o uso de semivariograma com a krigragem podem ser obtidos em Landin et al., 2002).

\subsection{Técnica dos Quantis}

A técnica dos quantis foi amplamente disseminada a partir dos estudos pioneiros realizados por Pinkayan (1966) que tinham como principal objetivo analisar a ocorrência de anos 
secos e chuvosos em extensas áreas continentais, especialmente nos Estados Unidos da América. As ordens quantílicas (p) propostas por Pinkayan (1966) e, bastante difundidas por Xavier (1999), são de $15 \% ; 35 \%$; 65\% e $85 \%$, que correspondem aos níveis ou categorias: Muito Seco (MS), Seco (S), Normal (N), Chuvoso (C) e Muito Chuvoso (MC), respectivamente. Xavier (1999) cita os quantis extremos, útil para analisar a ocorrência e frequência de extremos climáticos, com ordem quantílitas de 5\% e $95 \%$ referentes aos extremos de seca e chuva, respectivamente. Aqui adotou-se a nomenclatura Extremamente Seco (M.M. SECO) para a ordem quantílica de $\mathrm{p}=0,05 \mathrm{e}$, ii) Extremamente Chuvoso (M.M. CHUVOSO) para a ordem quantílica de $\mathrm{p}=0,95$.

\section{RESULTADOS E DISCUSSÃO}

As tendências dos índices de mudanças climáticas utilizados neste trabalho estão apresentados na Tabela 2, onde está indicado o sinal da tendência, bem como o seu nível de significância.

Neste caso, os valores em negrito correspondem às tendências com significância estatística ao nível de $5 \%(\mathrm{p} \leq 0,05)$ (alta) e os demais valores correspondem às tendências com significância estatística ao nível de $10 \%(\mathrm{p} \leq 0,10)$. Na Região Metropolitana do Recife (Ponto 1) os resultados diferem dos demais pontos por apresentar tendência negativa em praticamente todos os índices (exceção do DCS) indicando redução na precipitação, e alterações negativas na sua distribuição sazonal e intensidade, assim como, apresentou tendência de aumento nos dias consecutivos secos. Os índices precipitação total anual (PRCPTOT), dias consecutivos chuvosos (DCC) e dias consecutivos secos (DCS) apresentaram significância estatística em alguns dos pontos estudados. Nos localizados na mesorregião do Agreste Pernambucano observa-se tendência de aumento nos totais de precipitação. $\mathrm{O}$ ponto $2 \mathrm{a}$ foi o que apresentou os resultados mais significativos quando analisados todos os índices (8 dos 6 índices apresentaram alta significância).

A Figura 2 apresenta a variabilidade espacial das tendências dos índices PRCPTOT (a), DCC (b) e DCS (c).

Observa-se tendência negativa do índice na mesorregião da Região Metropolitana do Recife (RMR), Zona da Mata, passando a ficar positiva no agreste em direção ao sertão. Já o índice DCC apresentou tendência negativa em praticamente todo o estado, com exceção do sudoeste do Sertão do São Francisco. Por sua vez, o índice DCS apresentou tendência contrária (positiva) ao DCC. Os resultados são coerentes com os encontrados por Soares e Nóbrega (2009; 2010) com relação à ocorrência e tendência de veranicos na mesoregião do sertão pernambucano. Os autores verificaram através da análise da sequência de cinco ou mais dias secos dentro do período chuvoso (método usado pelos autores para definição de veranicos) que o número destes eventos tem aumentado, corroborando com as informações obtidas na análise de tendência de DCC, que, com a exceção do extremo sudoesteoeste do São Francisco Pernambuco, apresenta tendência de diminuição dos dias consecutivamente chuvosos, ou um aumento de dias consecutivamente secos, que se persistir por 5 dias ou mais, será um veranico, caso esta sequência de dias ocorra na estação chuvosa.

A Figura 3 mostra o comportamento espacial dos eventos extremos de chuvas da região estudada, tais como, os índices número de dias com chuvas acima de $50 \mathrm{~mm}$ (R50mm) (Figura 3a), quantidade máxima de precipitação em um dia (Rx1dia) (Figura 3b), quantidade máxima de precipitação em cinco dias consecutivos (Rx5dias) (Figura 3c), dias muito chuvosos (R95p) (Figura 3d) e dias extremamente chuvosos (R99p) (Figura 3e), respectivamente. Na maior parte do estado, há tendência de aumento de ocorrência de eventos extremos, com exceção da região da Zona da Mata, que apresenta tendência de redução.

Tabela 2: Tendências dos índices de extremos de precipitação para os 8 pontos analisados no estado de Pernambuco. Os valores em negrito correspondem às tendências com significância estatística ao nível de $5 \%(\mathrm{p} \leq 0,05)$ (alta) e os demais valores correspondem às tendências com significância estatística ao nível de $10 \%(\mathrm{p} \leq 0,10)$.

\begin{tabular}{|c|c|c|c|c|c|c|c|c|}
\hline & $\begin{array}{c}\text { Rx1dia } \\
\text { (mm/dia) }\end{array}$ & $\begin{array}{c}\text { Rx5dias } \\
\text { (mm/dia) }\end{array}$ & $\begin{array}{c}\text { R50mm } \\
\text { (dias/ano) }\end{array}$ & $\begin{array}{c}\text { R95p } \\
\text { (dias/ano) }\end{array}$ & $\begin{array}{c}\text { R99p } \\
\text { (dias/ano) }\end{array}$ & $\begin{array}{c}\text { DCC } \\
\text { (dias/ano) }\end{array}$ & $\begin{array}{c}\text { DCS } \\
\text { (dias/ano) }\end{array}$ & $\begin{array}{c}\text { PRCPTOT } \\
\text { (mm/ano) }\end{array}$ \\
\hline 1 & - & - & - & - & - & $\mathbf{- 0 , 6 7 5}$ & 0,238 & $-18,536$ \\
\hline 2a & $\mathbf{0 , 8 7 5}$ & $\mathbf{1 , 2 0 6}$ & $\mathbf{0 , 0 1 5}$ & $\mathbf{6 , 0 9 1}$ & $\mathbf{3 , 3 8 9}$ & $\mathbf{- 0 , 3 0 2}$ & - & - \\
\hline 2b & $\mathbf{0 , 9 5}$ & - & $\mathbf{0 , 0 2 8}$ & $\mathbf{8 , 7 2 3}$ & $\mathbf{5 , 1 3 7}$ & $-0,379$ & - & $\mathbf{1 0 , 8 0 8}$ \\
\hline 3a & $\mathbf{0 , 5 5 9}$ & - & 0,033 & $\mathbf{7 , 0 0 8}$ & $\mathbf{3 , 2 6 3}$ & $\mathbf{- 0 , 2 9 4}$ & - & - \\
\hline 3b & $\mathbf{0 , 6 2 4}$ & $\mathbf{0 , 7 5 1}$ & 0,02 & $\mathbf{6 , 0 4 7}$ & $\mathbf{2 , 5 2 1}$ & $\mathbf{- 0 , 2 6 7}$ & - & - \\
\hline 3c & $\mathbf{0 , 8 9 2}$ & - & $\mathbf{0 , 0 4 8}$ & $\mathbf{5 , 1 1 4}$ & $\mathbf{3 , 8 5 4}$ & - & - & - \\
\hline 4a & - & - & - & $\mathbf{4 , 5 1 6}$ & 1,952 & $\mathbf{- 0 , 2 7 7}$ & - & - \\
\hline 4b & $\mathbf{0 , 6 9 3}$ & 1,014 & 0,017 & 2,863 & $\mathbf{2 , 1 8 3}$ & - & $\mathbf{- 0 , 9 8 4}$ & - \\
\hline
\end{tabular}




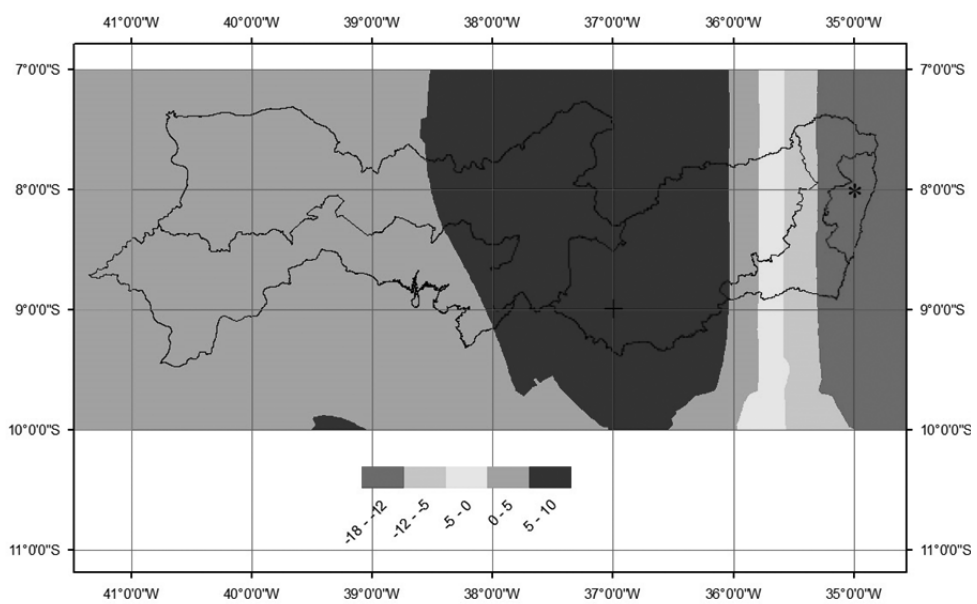

a)

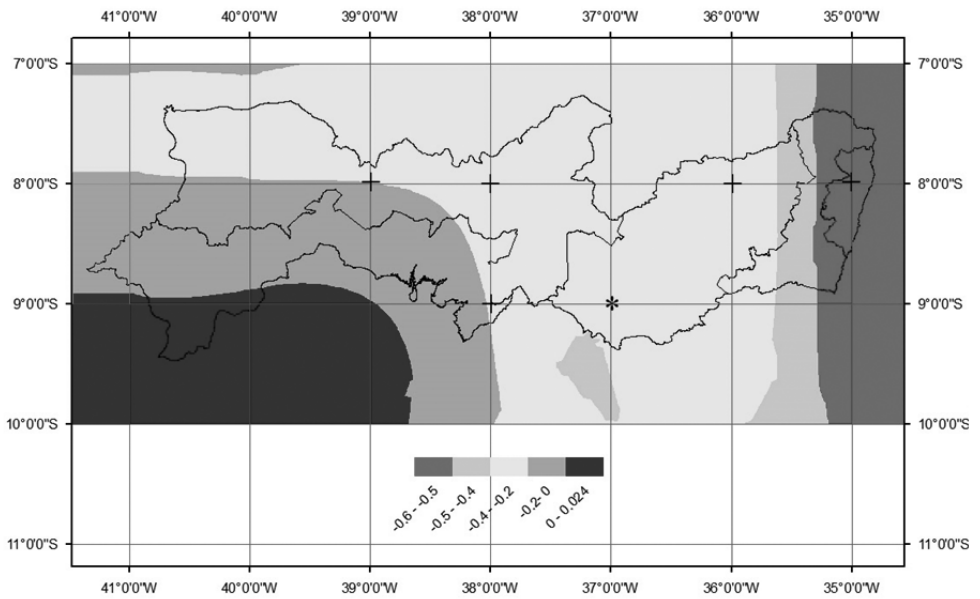

b)

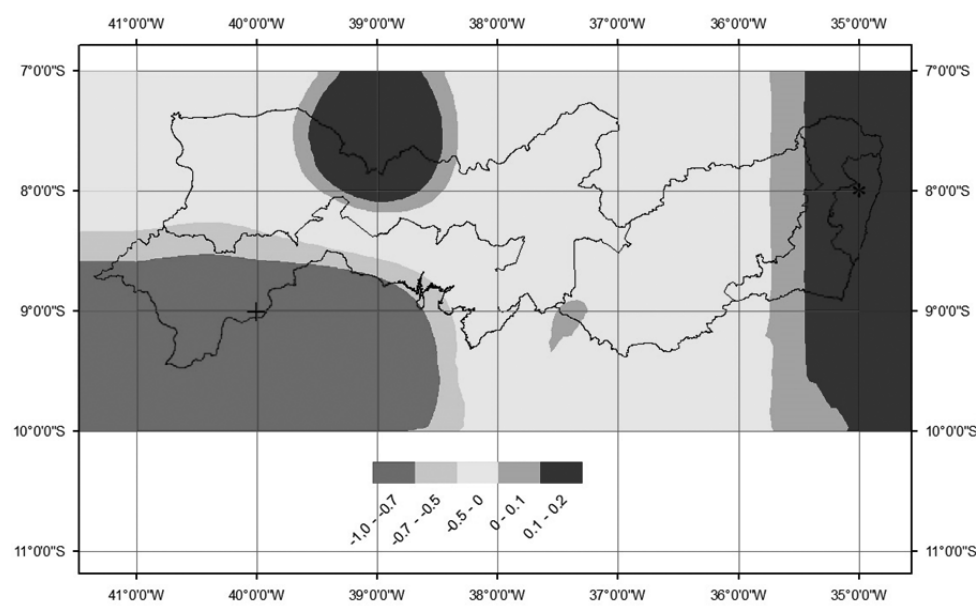

c)

Figura 2 - Variabilidade espacial dos índices Precipitação Total Anual (PRCPTOT) (a), Dias Consecutivos Chuvosos (DCC) (b) e Dias Consecutivos Secos (DCS) (c) (pontos de grade com + correspondem às tendências com significância estatística ao nível de 5\% (p $\leq 0,05)($ alta) e com * correspondem às tendências com significância estatística ao nível de $10 \%(\mathrm{p} \leq 0,10))$. 


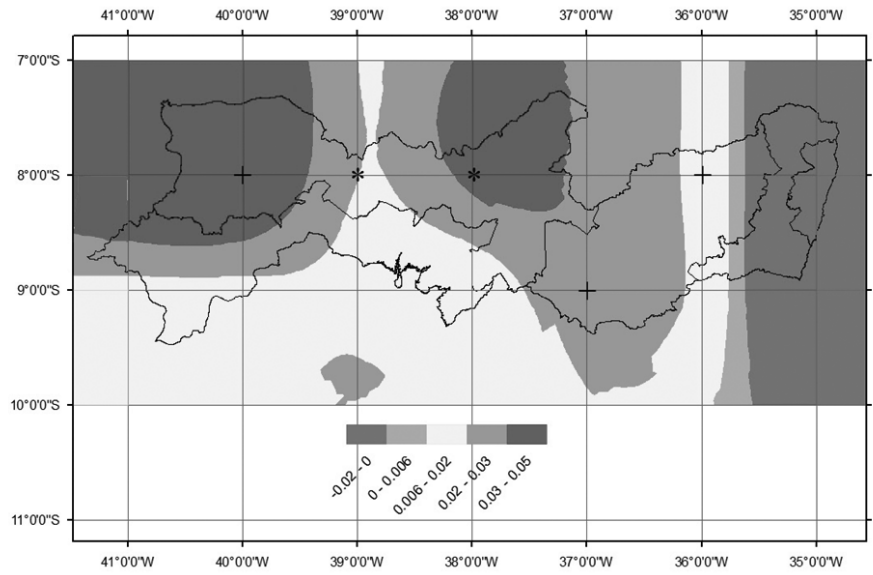

a)

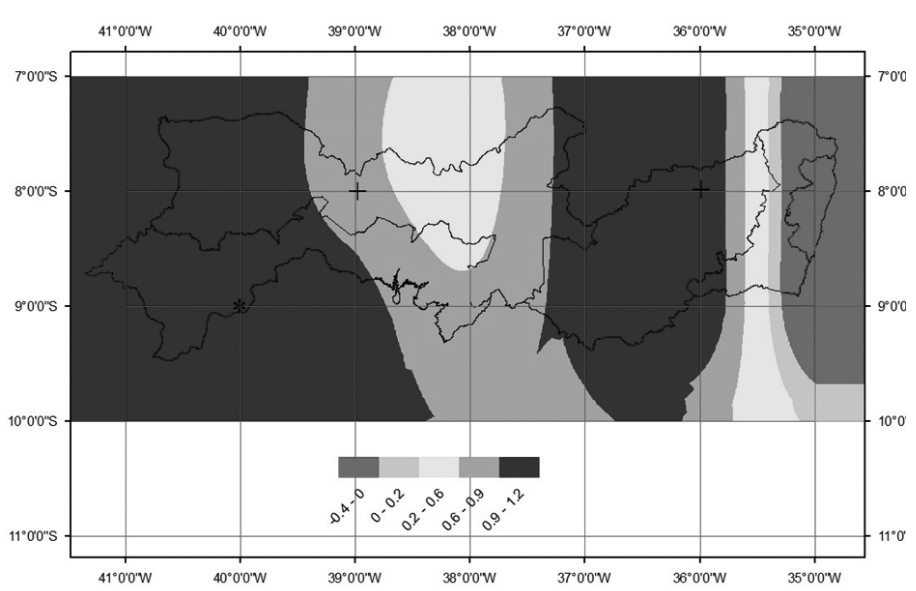

c)

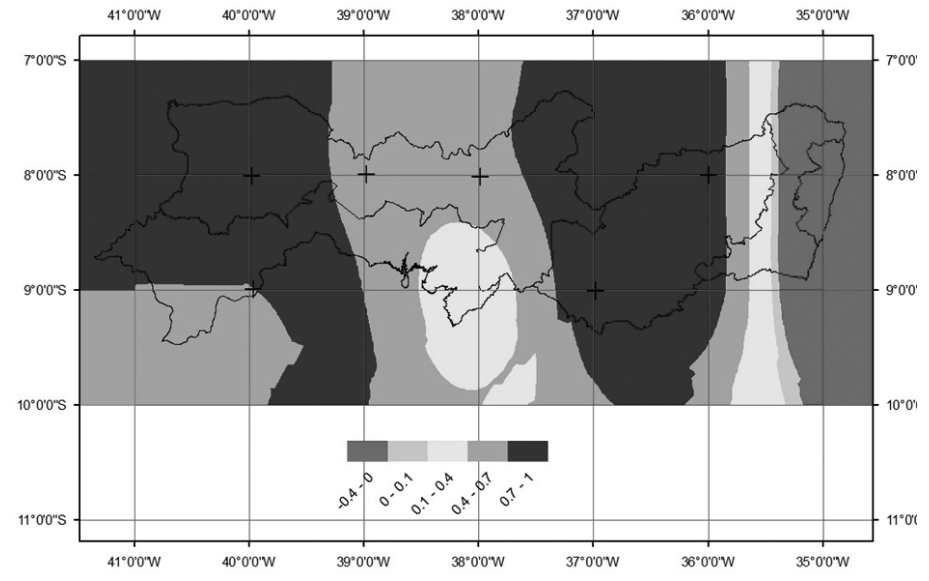

b)

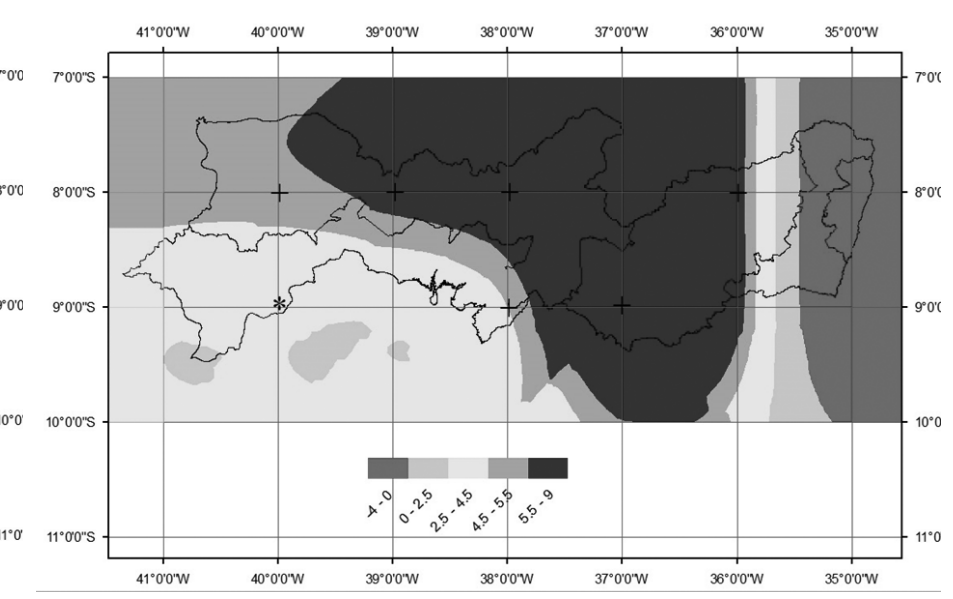

d)

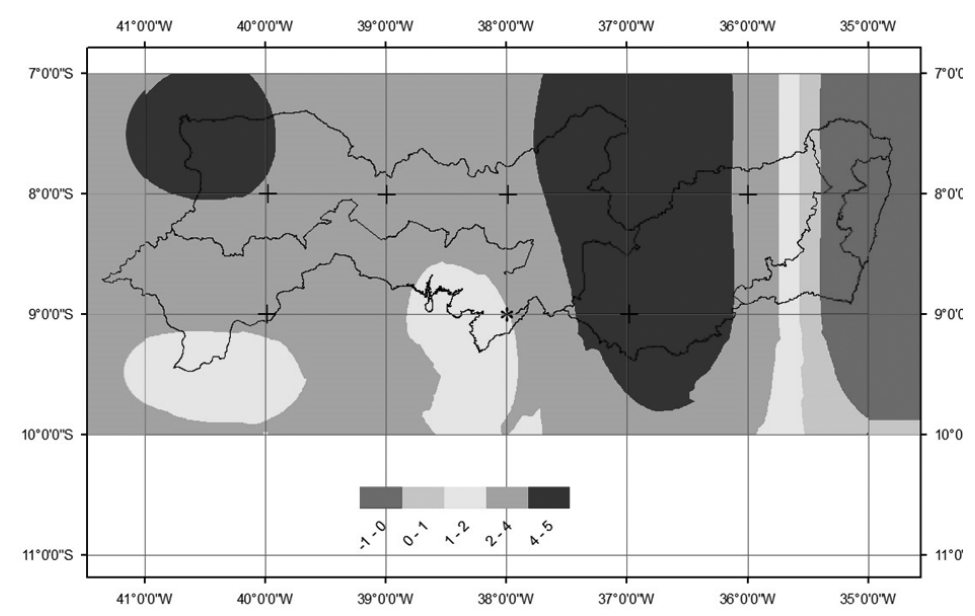

e)

Figura 3 - Variabilidade espacial dos índices Número de dias com chuva acima de 50mm (R50mm) (a), Quantidade máxima de precipitação em um dia (Rx1dia) (b), Quantidade máxima de precipitação em cinco dias consecutivos (Rx5dias) (c), Dias muito chuvosos (R95p) (d), e Dias muito secos (R99p) (e) (pontos de grade com + correspondem às tendências com significância estatística ao nível de $5 \%(\mathrm{p} \leq 0,05)$ (alta) e com * correspondem às tendências com significância estatística ao nível de 10\% $(\mathrm{p} \leq 0,10)$ ). 
Estes resultados corroboram com estudos anteriormente realizados para o Nordeste do Brasil (NEB), como no caso de Groisman et al. (2005) e Marengo (2007), nos quais identificaram tendência sistemática para aumento de chuva.

De modo geral, pode-se dizer que há tendência negativa em todos estes índices na RMR, enquanto que nas demais mesorregiões há tendência positiva. Estes resultados indicam que as chuvas extremas estão se tornando menos frequentes da mesorregião da RMR e mais frequentes nas demais partes do estado (índice R50mm), incluindo a tendência positiva no volume de chuva nestas regiões, uma vez que os índices Rx1dia e Rx5dias apresentaram este comportamento descrito. De fato, se a quantidade máxima de chuva em um dia e 5 dias consecutivos apresentam tendência positiva, os volumes de precipitação total e/ou mensal podem aumentar.

Os resultados, como um todo, indicam modificações nos volumes acumulados anuais de chuva. Uma vez que o clima está presente no cotidiano da sociedade e influencia diretamente a maioria de suas ações, estas modificações podem implicar em impactos nas atividades sociais, econômicas e até culturais. Os índices aqui analisados, como o DCC e DCS, evidenciam que a precipitação está se concentrado em curto período de tempo nas mesorregiões do agreste, sertão e sertão do São Francisco. Na RMR e parte da Zona da Mata (parte leste), onde há grande concentração urbana, há diminuição dos dias consecutivos chuvosos, acarretando em aumento dos dias consecutivos secos, assim como, na redução do total anual de precipitação.

Estas duas mesorregiões são representativas do ponto de vista de produção agrícola, sobretudo através da produção sulcroalcooleira, diretamente influenciada por variações sazonais na precipitação. Há algum tempo tem sido observado acentuado crescimento de produção industrial em áreas de produção de cana-de-açúcar, culminando também, como descrito em Campos et al. (2007), com momentos de queda ou estagnação desde o início da década de 1990. Tendências climáticas negativas nos dias consecutivos chuvosos influenciam na produção agrícola, podendo produzir entressafras maiores, e com isso a força de trabalho, que na zona da mata pernambucana é local, tende a procurar outras atividades empregatícias.

Já sobre os sertões, as chuvas têm aumentado de volume anual, no entanto estão ocorrendo menos dias consecutivos chuvosos (estão menores), mais dias consecutivos secos, (em maior número) e há concentração de volumes significativos de precipitação em poucos dias, ou seja, apesar do volume total anual de chuva estar maior, a distribuição temporal está com tendência negativa. Sabe-se que a precipitação média anual no sertão pernambucano pode ser maior que $500 \mathrm{~mm}$ em muitas localidades, no entanto, a sua distribuição temporal está concentrada em 3 meses, e com base nestes resultados, pode-se dizer que as chuvas estão cada vez mais concentradas. Este fato é preocupante em alguns aspectos, como por exemplo, na produção agrícola, pois se não houver armazenamento adequado de água, ela se tornará mais escassa. Em contrapartida, se bem planejado, a captação de água pode ser maior, sendo uma medida importante para manutenção de água na região (Oliveira et al., 2012).

A partir da Tabela 3 pode-se ter uma noção das características pluviométricas do Estado de Pernambuco, a respeito dos totais pluviométricos e de sua distribuição ao longo ano, e observar as categorias de ordem quantílica (Qp) extremamente secos $(\mathrm{Qp}=0,05)$ e extremamente chuvosos $(\mathrm{Qp}=0,95)$, segundo a metodologia descrita por Xavier et al. (2007), para cada mês.

Os maiores volumes médios de precipitação, bem como, os máximos e mínimos ocorrem no ponto 1 , que representa a Região Metropolitana do Recife e Zona da Mata. Os volumes diminuem na direção oeste, assim como a distribuição sazonal também é modificada. Nos pontos de 1 a 2 (Região Metropolitana, Zona da Mata e Agreste) as chuvas são mais volumosas entre o outono e inverno, enquanto nos pontos 3 a 4 as chuvas são mais volumosas do verão a outono.

Com relação à frequência de ocorrência dos eventos extremamente secos e chuvosos, pode-se observar na Tabela 4, que a mesorregião da Região Metropolitana do Recife (1) foi a que demonstrou maior regularidade de ocorrência dos eventos extremamente secos e chuvosos, apresentando o predomínio de dois episódios para cada mês ao longo dos 32 anos de observação, apenas o mês de janeiro que apresentou um episódio de evento extremamente seco.

O Agreste pernambucano apresentou o predomínio de ocorrência de eventos extremamente negativos $(\mathrm{Qp}=0,05) \mathrm{em}$ ambas as áreas ( $2 a$ e $2 b)$, já para os eventos positivos foram apenas quatro episódios, sendo dois na localidade 2a (um no mês de janeiro e um no mês de novembro) e dois na localidade $2 b$ (no mês de janeiro).

As mesorregiões do Sertão Pernambucano e do São Francisco foram as que apresentaram maior variabilidade com relação à ocorrência de meses extremamente seco e chuvosos. O Sertão Pernambucano apresentou predomínio de ocorrência de eventos negativos (M.M.SECO), quando comparado com os eventos positivos (M.M.CHUVOSOS), sendo 52 eventos extremamente secos distribuídos entre as localidades $3 \mathrm{a}, 3 \mathrm{~b}$ e $3 \mathrm{c}$, e 35 episódios de eventos extremamente chuvosos ao longo de 32 anos de dados. Foi encontrado que existe regularidade durante todo ano no número de ocorrência de eventos extremamente secos (duas ocorrências), já para a ocorrência dos eventos positivos, observa-se uma predominância de episódios concentrados nos meses de dezembro, janeiro e fevereiro (verão), com destaque para o ponto $3 \mathrm{c}$ que apresentou 
Tabela 3 - Variabilidade sazonal da precipitação nas Mesorregiões do Estado de Pernambuco, com os limites mensais de precipitação baseado nas ordens quantílicas extremamente secos $(\mathrm{Q} 0,05)$ e extremamente chuvosos $(\mathrm{Q} 0,95)$.

\begin{tabular}{|c|c|c|c|c|c|c|c|c|c|c|c|c|c|}
\hline & & Jan & Fev & Mar & Abr & Mai & Jun & Jul & Ago & Set & Out & Nov & Dez \\
\hline \multirow{5}{*}{1} & Med & 89 & 122 & 192 & 251 & 279 & 353 & 284 & 183 & 93,8 & 51,3 & 45,7 & 48,7 \\
\hline & Max & 204 & 351 & 443 & 558 & 526 & 620 & 593 & 417 & 347 & 142 & 256 & 172 \\
\hline & Mín & 24 & 11 & 47 & 51 & 36 & 100 & 106 & 24 & 12 & 9 & 4 & 4 \\
\hline & Q0,05 & 30 & 30 & 60 & 72 & 89 & 144 & 142 & 85 & 25 & 16 & 9 & 7 \\
\hline & Q0,95 & 174 & 241 & 402 & 486 & 451 & 572 & 550 & 343 & 219 & 100 & 79 & 142 \\
\hline \multirow{5}{*}{$2 a$} & Med & 41 & 61 & 90 & 98 & 90 & 124 & 108 & 69 & 38 & 17 & 14 & 23 \\
\hline & Max & 266 & 230 & 300 & 261 & 185 & 308 & 189 & 164 & 207 & 117 & 3 & 98 \\
\hline & Mín & 1 & 0 & 3 & 11 & 8 & 25 & 33 & 21 & 7 & 1,9 & 0 & 1 \\
\hline & $\mathrm{Q} 0,05$ & 8 & 6 & 23 & 23 & 26 & 48 & 51 & 25 & 9 & 2 & 2 & 1 \\
\hline & Q0,95 & 105 & 160 & 189 & 188 & 178 & 230 & 170 & 129 & 107 & 42 & 42 & 75 \\
\hline \multirow{5}{*}{$2 b$} & Med & 44 & 59 & 86 & 86 & 75 & 99 & 83 & 52 & 28 & 18 & 61 & 28 \\
\hline & Max & 281 & 161 & 238 & 268 & 188 & 426 & 151 & 108 & 98 & 84 & 61 & 128 \\
\hline & Mín & 2 & 3 & 0 & 5 & 2 & 23 & 27 & 8 & 1 & 0 & 0 & 0 \\
\hline & $\mathrm{Q} 0,05$ & 5 & 5 & 7 & 12 & 12 & 29 & 31 & 14 & 5 & 1 & 0 & 1 \\
\hline & Q0,95 & 68 & 143 & 179 & 215 & 163 & 174 & 127 & 101 & 77 & 72 & 38 & 96 \\
\hline \multirow{5}{*}{$3 a$} & Med & 92 & 116 & 159 & 134 & 87 & 48 & 34 & 16 & 12 & 16 & 20 & 39 \\
\hline & Max & 426 & 329 & 362 & 316 & 345 & 155 & 61 & 57 & 91 & 119 & 69 & 138 \\
\hline & Mín & 4 & 14 & 14 & 12 & 0 & 0 & 2 & 1 & 0 & 0 & 0 & 0 \\
\hline & Q0,05 & 17 & 23 & 37 & 34 & 4 & 6 & 6 & 2 & 0 & 0 & 0 & 3 \\
\hline & Q0,95 & 234 & 230 & 320 & 282 & 212 & 136 & 59 & 44 & 38 & 57 & 62 & 115 \\
\hline \multirow{5}{*}{$3 b$} & Med & 119 & 133 & 171 & 123 & 49 & 22 & 6 & 6 & 11 & 14 & 24 & 63 \\
\hline & Max & 496 & 305 & 285 & 297 & 274 & 83 & 119 & 33 & 184 & 120 & 144 & 197 \\
\hline & Mín & 20 & 23 & 52 & 21 & 0 & 0 & 1 & 0 & 0 & 0 & 3 & 2 \\
\hline & Q0,05 & 29 & 43 & 61 & 26 & 2 & 3 & 2 & 0 & 0 & 0 & 3 & 11 \\
\hline & Q0,95 & 241 & 276 & 268 & 258 & 95 & 70 & 48 & 26 & 21 & 55 & 68 & 171 \\
\hline \multirow{5}{*}{$3 c$} & Med & 120 & 123 & 166 & 123 & 41 & 14 & 18 & 3 & 13 & 14 & 28 & 75 \\
\hline & Max & 412 & 360 & 320 & 470 & 137 & 53 & 42 & 9 & 287 & 96 & 124 & 25 \\
\hline & Mín & 20 & 7 & 37 & 11 & 1 & 1 & 1 & 0 & 0 & 0 & 2 & 6 \\
\hline & $\mathrm{Q} 0,05$ & 25 & 44 & 72 & 19 & 4 & 2 & 2 & 0 & 0 & 0 & 3 & 14 \\
\hline & $\mathrm{Q} 0,95$ & 234 & 267 & 302 & 314 & 113 & 36 & 32 & 8 & 15 & 45 & 65 & 243 \\
\hline \multirow{5}{*}{$4 a$} & Med & 57 & 63 & 110 & 73 & 52 & 50 & 46 & 23 & 13 & 14 & 22 & 42 \\
\hline & Max & 206 & 198 & 340 & 228 & 315 & 165 & 115 & 77 & 407 & 85 & 73 & 259 \\
\hline & Mín & 2 & 7 & 0 & 1 & 2 & 5 & 13 & 1 & 0 & 0 & 0 & 1 \\
\hline & $\mathrm{Q} 0,05$ & 5 & 9 & 11 & 4 & 4 & 12 & 15 & 4 & 0 & 0 & 1 & 2 \\
\hline & Q0,95 & 175 & 176 & 207 & 219 & 129 & 107 & 94 & 55 & 31 & 69 & 68 & 103 \\
\hline \multirow{5}{*}{$4 b$} & Med & 91 & 78 & 115 & 71 & 22 & 10 & 9 & 3 & 10 & 14 & 30 & 57 \\
\hline & Max & 448 & 246 & 233 & 211 & 106 & 51 & 43 & 16 & 234 & 79 & 138 & 288 \\
\hline & Mín & 6 & 3 & 14 & 0 & 0 & 0 & 1 & 0 & 0 & 0 & 0 & 4 \\
\hline & $\mathrm{Q} 0,05$ & 11 & 8 & 23 & 9 & 0 & 0 & 1 & 0 & 0 & 0 & 0 & 8 \\
\hline & Q0,95 & 315 & 191 & 220 & 194 & 76 & 31 & 26 & 8 & 15 & 73 & 97 & 131 \\
\hline
\end{tabular}

Legendas: Med - Precipitação média; Max - Precipitação máxima média, Mín - Precipitação mínima média; Q0,05 - Quantil 5\%, referente a extremamente seco; Q0,95 - Quantil 95\%, referente a extremamente chuvoso.

a maior ocorrência de eventos positivos em um único mês (sete episódios no mês de janeiro). Este resultado encontrado condiz com o período chuvoso no Sertão Pernambucano, que é entre os meses de verão/outono (Kousky, 1980; Soares e Nóbrega, 2010; Farias et al., 2012).
O Sertão do São Francisco apresentou resultados semelhantes à mesorregião do Sertão Pernambucano, mostrando o predomínio de ocorrência de eventos extremamente secos, destaque para localidade $4 \mathrm{a}$, que apresentou a maior ocorrência de eventos negativos, total de 33 episódios. Enquanto que 
Tabela 4 - Número de ocorrência de eventos nos meses extremamente secos $(\mathrm{Q}(\mathrm{p})=0,05)$ e extremamente chuvosos $(\mathrm{Q}(\mathrm{p})=0,95)$ nas $\mathrm{Mes}$ (rregiões do Estado de Pernambuco.

\begin{tabular}{|c|c|c|c|c|c|c|c|c|c|c|c|c|c|}
\hline Área & $Q(p)$ & Jan & Fev & Mar & Abr & Mai & Jun & Jul & Ago & Set & Out & Nov & Dez \\
\hline \multirow{2}{*}{1} & 0,05 & 1 & 2 & 2 & 2 & 2 & 2 & 2 & 2 & 2 & 2 & 2 & 2 \\
\hline & 0,95 & 2 & 2 & 2 & 2 & 2 & 2 & 2 & 2 & 2 & 2 & 2 & 2 \\
\hline \multirow{2}{*}{$2 a$} & 0,05 & 2 & 2 & 2 & 2 & 2 & 2 & 2 & 2 & 2 & 0 & 2 & 0 \\
\hline & 0,95 & 1 & 0 & 0 & 0 & 0 & 0 & 0 & 0 & 0 & 0 & 1 & 0 \\
\hline \multirow{2}{*}{$2 b$} & 0,05 & 2 & 2 & 2 & 2 & 2 & 2 & 2 & 2 & 2 & 0 & 2 & 0 \\
\hline & 0,95 & 2 & 0 & 0 & 0 & 0 & 0 & 0 & 0 & 0 & 0 & 0 & 0 \\
\hline \multirow{2}{*}{$3 a$} & 0,05 & 2 & 2 & 2 & 2 & 2 & 2 & 2 & 2 & 0 & 0 & 0 & 2 \\
\hline & 0,95 & 3 & 1 & 0 & 0 & 0 & 0 & 0 & 0 & 0 & 1 & 0 & 0 \\
\hline \multirow{2}{*}{$3 b$} & 0,05 & 2 & 2 & 2 & 2 & 2 & 2 & 2 & 0 & 0 & 0 & 0 & 2 \\
\hline & 0,95 & 4 & 4 & 0 & 0 & 0 & 0 & 0 & 0 & 0 & 1 & 1 & 4 \\
\hline \multirow{2}{*}{$3 c$} & 0,05 & 2 & 2 & 2 & 2 & 2 & 2 & 2 & 0 & 0 & 0 & 2 & 2 \\
\hline & 0,95 & 7 & 2 & 0 & 0 & 0 & 0 & 0 & 0 & 1 & 0 & 1 & 5 \\
\hline \multirow{2}{*}{$4 a$} & 0,05 & 7 & 5 & 6 & 10 & 1 & 1 & 0 & 0 & 0 & 0 & 0 & 3 \\
\hline & 0,95 & 2 & 0 & 0 & 1 & 0 & 0 & 0 & 0 & 0 & 0 & 0 & 1 \\
\hline \multirow{2}{*}{$4 b$} & 0,05 & 2 & 2 & 2 & 2 & 0 & 0 & 0 & 0 & 0 & 0 & 0 & 2 \\
\hline & 0,95 & 4 & 1 & 0 & 0 & 0 & 0 & 0 & 0 & 1 & 0 & 3 & 2 \\
\hline
\end{tabular}

os eventos extremamente chuvosos concentraram-se na mesorregião do Sertão Pernambucano no período chuvoso, dezembro, janeiro e fevereiro (verão).

Sugere-se que os resultados encontrados sejam aprofundados do ponto de vista de variabilidade climática interanual e intrasazonal, uma vez que as temperaturas da superfície dos oceanos Attântico e Pacífico tem papel primordial nas chuvas da área estudada, assim como no posicionamento de sistemas atmosféricos como a Zona de Convergência Intertropical, Ondas de Leste e Vórtices Ciclones de Altos Níveis, e que podem ajudar a estabelecer anos que se associem a maior ou menor frequência desses sistemas, e consequentemente explicar o aumento ou a diminuição de chuvas baseada na metodologia aqui utilizada.

\section{CONCLUSÕES}

A partir dos resultados encontrados é possível elaborar algumas considerações a respeito da climatologia de precipitação, tendo como base índices de extremos climáticos nas mesorregiões de Pernambuco.

Os resultados encontrados demonstraram a predominância de tendência de aumento no volume de chuva anual, com exceção da mesorregião da Região Metropolitana do Recife e parte leste da mesorregião da zona da mata, que apresentaram tendência negativa.

Com relação à ocorrência de eventos extremamente secos e chuvosos, segundo a metodologia dos quantis, pode-se destacar para o Estado de Pernambuco uma variação de ocorrência dos mesmos, onde cada região demonstra suas particularidades. Desta forma foi possível detectar para a mesorregião da Região Metropolitana certa regularidade de ocorrência para os episódios chuvosos e secos, enquanto que para as localidades do Agreste Pernambucano ocorreu a predominância de episódios extremamente secos.

O sertão de Pernambuco, representado pelas mesorregiões do Sertão Pernambucano e do São Francisco, foi que apresentou os maiores números de episódios extremamente secos, no entanto o que chama a atenção para o Sertão é a quantidade de ocorrência de eventos extremamente chuvosos, concentrados nos meses dezembro, janeiro e fevereiro, período chuvoso do Sertão de Pernambuco.

A partir dos estudos realizados é possível afirmar que vem ocorrendo mudanças nos padrões pluviométricos para o Estado de Pernambuco, porém não se pode afirmar que estas mudanças decorrem de fatores naturais ou fatores antrópicos.

\section{AGRADECIMENTOS}

Os autores agradecem a Fundação de Amparo a Ciência do Estado de Pernambuco (FACEPE) pela concessão da bolsa de Pós-Graduação Farias Lima, RP. Também agradecem apoio na infraestrutura física do Laboratório de Climatologia Tropical e Eventos Extremos (Tropoclima) através do Edital Multiusuários Propesq/UFPE. 


\section{REFERÊNCIAS}

CAMPOS, L. H. R.; RAPOSO, I; MAIA, A. Empregabilidade do cortador de cana-de-açúcar da zona da mata pernambucana no período de entressafra. Revista Econômica do Nordeste, v. 38, p. 329-342, 2007.

CHARBA J. P.; HARRELL, A. W.; LACKNER, A. C. A monthly precipitation amount climatology derived from published atlas maps: Development of a digital database. NOAA TDL Office Note 92-7, 20 pp. 1992.

COSTA, M. H.; FOLEY, J. A. Combined effects of deforestation and doubled atmospheric $\mathrm{CO}_{2}$ concentrations on the climate of Amazonia. Journal of Climate, v.13, p. 18-34, 2000.

FARIAS, R. F. L.; ALVES, K. M. A. S.; NÓBREGA, R. S. Climatologia de eocorrência de eventos extremos de precipitação na mesoregião do sertão pernambucano. Geonorte, v.1, n. 5, Ed. Especial 2, p. 930-941, 2012.

GLAHN, H. R.; CHAMBERS, T. L.; RICHARDSON, W. S.; PERROTTI, H. P. Objective map analysis for the local AFOS MOS Program. NOAA Technical Memorandum NWS TDL 75, NOAA, U. S. Department of Commerce, 34 pp., 1985. GROISMAN, P. Y.; KNIGHT, R. W.; EASTERLING, D. R.; KARL, T. R.; HEGERL, G. C.; RAZUVAEV, V. N. Trends in Intense Precipitation in the Climate Record. Journal of Climate, v. 18, p. 1326-1350, 2005.

HASTENRATH, S., GREISCHAR, L. Further Work on the Prediction of Northeast Brazil Rainfall Anomalies. Journal of Climate, v. 6, p. 743-758, 1993.

HAYLOCK, M. R., PETERSON, T. C., AlveS, L. M., AMBRIZZI, T., ANUNCIAÇÃO, Y. M. T., BAEZ, J., BARROS, V. R., BERLATO, M. A., BIDEGAIN, M., CORONEL, G., GARCIA, V. J., GRIMM, A. M., KAROLY, D., MARENGO, J. A., MARINO, M. B., MONCUNILL, D. F., NECHET, D., QUINTANA, J., REBELLO, E., RUSTICUCCI, M., SANTOS, J. L., TREBEJO, I., VINCENT, L. A. Trends in total and extreme South American rainfall 1960-2000 and links with sea surface temperature. Journal of Climate, v. 19, p. 1490-1512, 2006.

KOUSKY, V. Diurnal Rainfall Variation in Northeast Brazil. Monthly Weather Review, v. 108, p. 488-498, 1980.

LANDIN, P. M. B.; STURARO, J. R.; MONTEIRO, R. C. Exemplos de Aplicação de co-krigagem, UNESP / Campus de Rio Claro; Departamento de Geologia Aplicada - IGCE; Texto Didático - 09, 2002.

MARENGO, J. A. Caracterização do clima no Século XX e Cenários no Brasil e na América do Sul para o Século XXI derivados dos Modelos de Clima do IPCC. IN: Biodiversidade, 2006. 185 pág. 2007.
MAREnGO, J. A. Mudanças Climáticas Globais e seus efeitos sobre a Biodiversidade. IN: Biodiversidade 26 . Ministério do Meio Ambiente, 2006. 163 pág. 2006.

NIJSSEN, B.; O’DONNELL, G. M.; HAMLET, A. F.; LETTENMAIER, D. P. Hydrologic sensitivity of global rivers to climate change. Climatic Change, v. 50, p.143$175,2001$.

NÓBREGA, R. S.; SOUZA, E. P.; SOUZA, F. A. S. The impacts of changes in land cover on water resources in the western Amazon. Journal of Environmental Hydrology, v. 18, p. 1-12, 2010.

OLIVEIRA, G. C. S.; NÓBREGA, R. S.; ALMEIDA, H. A. Perfil socioambiental e estimativa do potencial para captação de água da chuva em Catolé de Casinhas, PE. Revista de Geografia, v. 29, p. 75-90, 2012.

PINKAYAN, S. Conditional probabilities of ocurrence of Wet and Dry Years Over a Large Continental Area. Colorado: State University, Boulder-Co, 1966.p. (Hidrology Papers, 12).

SANTOS, C. A. C.; BRITO, J. I. B. Análise dos índices de extremos para o semi-árido do Brasil e suas relações com TSM e IVDN. Revista Brasileira de Meteorologia, v. 22, p. 303-312, 2007.

SILVA, V. B. S.; KOUSKY, V. E.; SHI, W.; HIGGINS, R. W. An Improved Gridded Historical Daily Precipitation Analysis for Brazil. Journal of Hydrometeorology, v. 8, p. 847-861, 2007.

SOARES, D. B.; NÓBREGA, R. S. Análise espacial e climatológica da ocorrência de veranicos no Sertão de Pernambuco. Revista de Geografia, v. 27, p. 95-106, 2010.

SOARES, D. B.; NÓBREGA, R. S. Detecção de tendências na ocorrência de veranicos na microrregião do Pajeú - PE. Revista de Geografia, v. 26, p. 263-275, 2009.

XAVIER, T. M. B. S. Caracterização de períodos secos e ou excessivamente chuvosos no Estado do Ceará através da técnica dos quantis: 1964-1998. Revista Brasileira de Meteorologia, v. 14, n. 2, p. 63-78, 1999.

XAVIER, T. de M. B. S.; XAVIER, A. F. S.; ALVES, J. M. B. Quantis e eventos extremos: aplicações em ciências da terra e ambientais. Fortaleza: RDS, 2007. 277 p.

ZHANG, X., YANG, F. RClimDex (1.0) User Guide. Climate Research Branch Environment Canada. Downsview (Ontario, Canada), 2004, 22p. 